\title{
Lubrication mechanisms between parallel rough surfaces
}

\author{
Noël Brunetière • Arthur Francisco
}

Received: date / Accepted: date

\begin{abstract}
This paper presents a numerical analysis of the hydrodynamic pressure build-up between parallel surfaces. According to the Reynolds theory, it is not possible to generate a pressure between flat parallel sliding surfaces. However, experimental results demonstrate that mechanical seal surfaces can be separated by a full fluid film if the sliding speed is high enough. Several theories, including the roughness effect, have been proposed to explain this unexpected lift-off. A real demonstration of the roughness induced pressure generation has only recently been made possible with the development of deterministic mixed lubrication models. It is demonstrated that a transverse pumping mechanism that varies from place to place with the surface topography can explain the pressure build-up. A simplified model is proposed to include the transverse pumping and to simulate the roughness induced pressure generation.
\end{abstract}

Keywords Mixed lubrication · Hydrodynamic lubrication · Roughness

\section{Introduction}

In 1886, Reynolds [29] proposed an equation based on the the mass conservation of viscous fluid flows in thin films. The solution of this equation showed that it is possible to create a pressure between surfaces in relative motion if there are some height variations in the sliding direction. Using this theory, Reynolds has been able to explain the fluid pressure build up measured

\author{
N. Brunetière \\ Institut Pprime, CNRS, Université de Poitiers, Ensma, Futuroscope, France \\ Tel.: +33-549-496-531 \\ E-mail: noel.brunetiere@univ-poitiers.fr \\ F. Francisco \\ Institut Pprime, CNRS, Université de Poitiers, Ensma, Futuroscope, France \\ Tel.: +33-549-451-979 \\ E-mail: arthur.francisco@univ-poitiers.fr
}


by Beauchamp-Tower during its experiments on bearings. This equation has since been widely used with great success. According to this theory, it is not possible to generate a pressure if the surfaces are parallel (except in the case of a squeeze motion). However, there is considerable experimental evidence of pressure generation for this kind of configuration. These experiments mainly concern mechanical seals that are composed of two flat rings in relative motion [18]. Their flatness is usually within a few sodium light bands; that is, a fraction of microns. In the early-1960s, Denny [5] used pressure sensors to show that some hydrodynamic pressure is generated between the faces of a seal. In the same year, Summers-Smith [32] presented several friction tests that were performed on mechanical seals working in a range of pressure and speed with various fluids. When presented as a function of the duty parameter $G=\frac{\mu V\left(R_{o}-R_{i}\right)}{F}$, the friction exhibits a typical Stribeck shape with an initial decrease of the friction. After a threshold, the friction coefficient is a growing function of $G$, which indicates a hydrodynamic lubrication regime where the surfaces are fully separated by a lubricating film. This was confirmed by very low wear rate for this particular regime. The existence of a full fluid film between the parallel surfaces of a mechanical seal or flat-on-flat devices has been confirmed by several authors through friction curves [6], [20], [34], [30], [1] or film thickness measurements [26]. In 1987, Lebeck [16] reviewed the experiments that had been carried out on parallel sliding and highlighted the effect of roughness, viscosity, speed and other parameters. However, the experimental results did not give information on the mechanisms at the origin of the pressure build-up.

Nau dedicated two papers, in 1964 [24] and 1967 [23], to the analysis of the mechanisms that could explain the hydrodynamic pressure build-up, which are mainly based on surface flatness breakdown such as misalignment, waviness or roughness. To analyze the possibility of micro-lubrication due to roughness, Hamilton et al. [10] performed several tests on artificially textured roughness and found a pressure generation. However, their configuration was closer to what is now called surface texturing [9] rather than random roughness. Lebeck [17] identified four other possible mechanisms, including thermal wedge, viscosity wedge and squeeze effect between the asperities. According to Lebeck, the pressure build-up was actually due to a combination of these mechanisms. However, it did not offer a possibility to develop a simple model for the pressure generation. Accordingly, Lebeck used an empirical model based on experimental data: knowing the $G$ value, he could estimate the generated load [19]. So and Chen [31] proposed a theory based on micro-wedge lubrication that gave good results for flat-on-flat lubrication with a fluid feeding in the sliding direction as a slider bearing, where each asperity is a micro-slider bearing. However, this theory was not checked in the case of lateral fluid feeding as in the ring-on-flat or mechanical seal configuration.

It is not possible to simulate pressure generation between nominally parallel surfaces by applying initial averaging models for rough surfaces lubrication, such as flow factors theory $[28,27,33,12]$ or homogenization $[2,15,3]$. The increase of computational power has made it possible to handle fine simulations 
of the lubrication between parallel rough surfaces. Minet et al. [21] performed several simulations of the flows between the faces of a mechanical seal using a deterministic approach. For this task, the Reynolds equation, coupled with a cavitation algorithm, was solved on a very fine mesh that is able to capture the surface topography. These authors have been able to reproduce the experimentally obtained Stribeck curves. Their simulations also show that the roughness effect is high enough to generate a hydrodynamic lift able to separate the surfaces. The same authors [22] conducted parametric studies to highlight the impact of the pressure, load, viscosity, etc on the seal performance. However, even if an attempt has been made to introduce non-dimensional numbers to scale the results [4], the mechanisms have not been yet explained. Using numerical simulations applied to a mechanical seal, we will demonstrate that a transverse pumping mechanism that varies from place to place with the surface topography can explain the pressure build-up. A simplified model is then proposed to include the transverse pumping and to simulate the roughness induced pressure generation.

\section{Simulation}

\subsection{Numerical model}

The numerical model that has been used in the present work has been presented in previous papers $[21,22]$ but its main characteristics will be briefly recalled here. The configuration is given in figure 1. A smooth ring is rotating at constant speed while a static rough ring is placed at a given distance $h_{0}$. The configuration is thus stationary. A pressurized fluid is located at the outer radius and can flow through the gap to the inner radius, which is at atmospheric pressure. The distance between $h_{0}$ the two rings is calculated to ensure a balance between a closing force $F$ and the opening force due to the fluid pressure and the possible asperity contact.

A very fine mesh, whose cell size is about $1 \times 1 \mu \mathrm{m}^{2}$, is used to capture the surface topography. The Reynolds equation, including a mass-conserving cavitation algorithm, is solved with the finite volume method on this mesh:

$$
F_{s} \frac{\partial}{\partial r}\left(\frac{r h^{3}}{\mu} \frac{\partial D}{\partial r}\right)+F_{s} \frac{\partial}{\partial \theta}\left(\frac{h^{3}}{r \mu} \frac{\partial D}{\partial \theta}\right)=6 \omega r\left[\frac{\partial h}{\partial \theta}+\left(1-F_{s}\right) \frac{\partial h D}{\partial \theta}\right]
$$

In this equation, $h$ is the local film thickness, $\mu$ the fluid viscosity and $\omega$ the rotating speed. $D$ is a universal variable and $F_{s}$ a switch function for the cavitation algorithm:

$$
\left\{\begin{array} { r l } 
{ D } & { = p - p _ { c a v } } \\
{ \rho } & { = \rho _ { 0 } } \\
{ F _ { s } } & { = 1 }
\end{array} \quad \left\{\begin{array}{rl}
D & =\frac{\rho}{\rho_{0}}-1 \\
p & =p_{c a v} \\
F_{s} & =0
\end{array}\right.\right.
$$




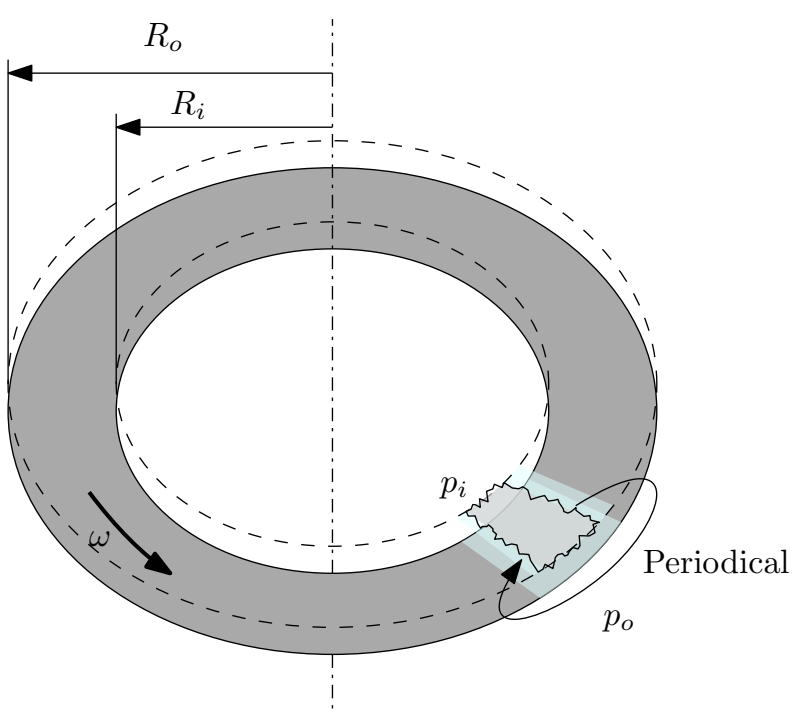

Fig. 1 Configuration of the problem

where $p_{\text {cav }}$ is the cavitation pressure and $\rho_{0}$ is the liquid density. Note that a multiscale version of the model [25] is used in the present work to reduce computation time.

Each summit of the rough surface in contact with the rotor is treated with an elliptical Hertzian model [11]. The friction force for each summit is the product of the calculated normal force by the contact friction coefficient $f_{c}$.

\subsection{Results}

The operating and design parameters of the studied mechanical seal are given in table 1. The fluid pressure and load are kept constant while the rotating speed is varied. Hence, the value of the duty parameter $G$ is defined as:

$$
G=\frac{\mu V\left(R_{o}-R_{i}\right)}{F}=\frac{\mu \omega\left(R_{o}^{2}-R_{i}^{2}\right)}{2 F}
$$

and varies from $2 \times 10^{-9}$ to $2 \times 10^{-7}$. The average sliding speed $V$ is equal to the product of the rotating speed $\omega$ and the mean radius $R_{m}=\frac{R_{o}-R_{i}}{2}$.

During the simulations, four statistically equivalent rough surfaces are used. Their characteristics are presented in table 2, while a top view of these surfaces is presented on figure 2. The statistical parameters used for the surfaces generationare:

- Roughness height $S q=\left(\frac{1}{N} \sum_{1}^{N} z_{i}^{2}\right)^{1 / 2}$

- Skewness parameter $S S k=\frac{1}{N S q^{3}} \sum_{1}^{N} z_{i}^{3}$

- Kurtosis parameter $S K u=\frac{1}{N S q^{4}} \sum_{1}^{N} z_{i}^{4}$ 
Table 1 Operating and design parameters of the seal

\begin{tabular}{lc}
\hline Parameter & Value \\
\hline Outer radius $R_{o}(m)$ & 0.033 \\
Inner radius $R_{i}(m)$ & 0.029 \\
Rotating speed $\omega\left({\left.\mathrm{rad} . s^{-1}\right)}^{-1}\right)$ & $10-900$ \\
Applied Force $F(N)$ & 584 \\
Outer pressure $p_{o}(M P a)$ & 1 \\
Inner pressure $p_{i}(M P a)$ & 0 \\
Cavitation pressure $p_{c a v}(M P a)$ & -0.01 \\
Fluid viscosity $\mu(P a . s)$ & 0.001 \\
Fluid density $\rho_{0}\left(\mathrm{~kg} . \mathrm{m}^{-3}\right)$ & 1000 \\
Friction coefficient between asperities $f_{c}$ & 0.2 \\
Composite elastic modulus of materials $E^{\prime}(G P a)$ & 39.7 \\
\hline
\end{tabular}

Table 2 Parameters of the rough surfaces used in the study

\begin{tabular}{lc}
\hline Parameter & Value \\
\hline Roughness height $S q(\mu m)$ & 0.1 \\
Skewness $S k$ & -2 \\
Kurtosis $K u$ & 30 \\
Radial correlation length at $80 \% \lambda_{r}(\mu m)$ & 8 \\
Circumferential correlation length at $80 \% \lambda_{\theta}(\mu m)$ & 10 \\
Radial number of points & 4096 \\
Circumferential number of points & 256 \\
Circumferential sampling at mid-radius $(\mu m)$ & 1 \\
\hline
\end{tabular}

- Auto-correlation function $A C F(x=n \Delta x, y=m \Delta y)=\frac{1}{N S q^{2}} \sum_{1}^{N} z_{i, j} z_{i+n, j+m}$

where $N$ is the number of grid points. Note that the mean height of the surface is 0 . The correlation length is measured from the center of the ACF to the point where its value has decreased to $80 \%$ of its initial value, which gives an indication on the typical lateral size of the roughness.

The Hu and Tonder method [14] is used for the surface generation. It allows, from a random Gaussian white noise to generate a random Gaussian surface with a given ACF and a given roughness height $S q$. To impose nonGaussian character, that is to say prescribed skewness and kurtosis values, the Hill et al. [13] algorithm coupled with the Watson and Spedding method [35] are used. Even if these methods are efficient, it is well know that they lead to some deviations from the prescribed parameters [7]. The values of the exact parameters of the 4 surfaces are given in table 3 . It can be seen that the deviation on $S k$ and $K u$ is less than $3 \%$ of the prescribed values which ensures statistical equivalence between the 4 surfaces. In addition, the maximum peak height and valley depth are indicated. It should be noted that the surfaces were thresholded at $5 S q$ to avoid any unrealistic contact despite high film thickness. In the present case, a maximum of 50 points (over a total of 1 million points) per surface were truncated. This thresholding has thus no impact on the surfaces statistics.

Figure 3 presents the variation of the average distance between the seal surfaces when the duty parameter $G$ is varied. For each surface, there is a 
Table 3 Real parameters of the rough surfaces used in the study

\begin{tabular}{lcccc}
\hline Surface number & 1 & 2 & 3 & 4 \\
\hline Skewness $S k$ & -1.97 & -1.95 & -2.05 & -1.96 \\
Kurtosis $K u$ & 29.89 & 29.76 & 30.16 & 29.78 \\
Maximum peak height $S p / S q$ & 5.0 & 5.0 & 4.04 & 3.85 \\
Maximum valley depth $S v / S q$ & -44.4 & -48.2 & -49.8 & -51.3 \\
\hline
\end{tabular}
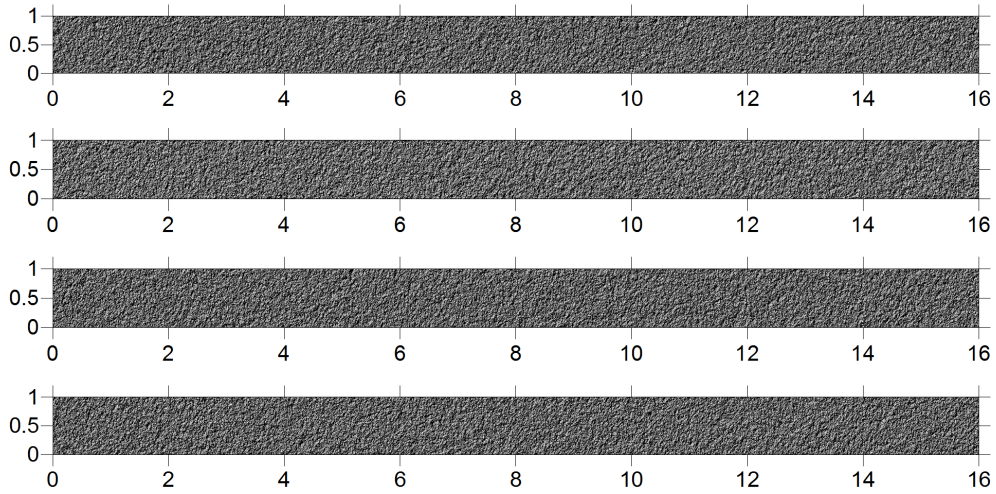

Fig. 2 Rough surfaces used in the simulations: from top to bottom, surface 1 to 4

plateau, or at least a low slope variation, at low $G$ values and then an increase of the film thickness due to the roughness induced hydrodynamic pressure. The $G$ value corresponding to the end of the plateau changes from one surface to the other. Note that only surface 1 exhibits a reverse evolution (i.e. decrease in the film thickness when $G$ increases) at high $G$ values which is probably correlated with a rapid spread of cavitation area leading to a decrease in hydrodynamic force.

Figure 4 shows the ratio of the asperities contact force $F_{c}$ normalized by the total load $F$ as a function of the $G$ value. It appears that the film thickness plateau is correlated to the occurrence of solid contact. The film thickness starts to increase more rapidly when the contact force vanishes. Note that only surfaces 2, 3 and 4 reach the full hydrodynamic lubrication regime.

The apparent friction coefficient is presented as a function of the duty parameter for each surface on figure 5. Typical Stribeck curves are obtained. The friction coefficient first decreases when $G$ is raised, which is typical of the mixed lubrication regime. A minimum is then reached and the friction coefficient starts to increase with the duty parameter. The beginning of the full hydrodynamic regime is located after this minimum. As discussed by Brunetière et al. [4], a slope of 0.75 is obtained on the friction curve when the surfaces are fully separated. For surface 1, which exhibits high asperity contact, the typical Stribeck curve is not observed.

Even if the 4 surfaces have the same statistical roughness parameters, they exhibit different behavior in terms of friction level as can be seen in figure figure 5 . These differences that can be surprising are however correlated with 
experimental findings [22]. The origin of the variation in friction performance is due to:

1. Difference in asperities contact force;

2. Difference in hydrodynamic load carrying capacity.

Concerning the contact, it should be keep in mind that it is a slight contact level with less than $1 \%$ of points in contact in the worst case (surface 1 at lowest $G$ values). Thus only the top asperities experience contact. The maximum peak height value $S p$ that controls that distance at which contact will occur will thus have an impact. The value of the contact force (presented in figure 4) is indeed well correlated, at low $G$ values, with the $S p$ value of each surface (see table 3 ). However, when comparing the results of surface 1 and 2 having the same $S p$ value but significantly different contact force for the same surface surface distance, it is clear that $S p$ is not the only parameters. The contact force will certainly also depend on the other few highest summits which are different from one surface to the other. This contact mechanics problem is out of topic of the present paper and will not be addressed here. The most interesting point is the difference in hydrodynamic load generation. Since this force is generated by the whole surface and not only the highest summits, it is less sensitive to the surface than the contact force. Indeed, at the highest $G$ value, for which all the surfaces are almost in full hydrodynamic regime, the difference in friction between the four surfaces is significantly reduced. This point will be analyzed in more depth in this paper.

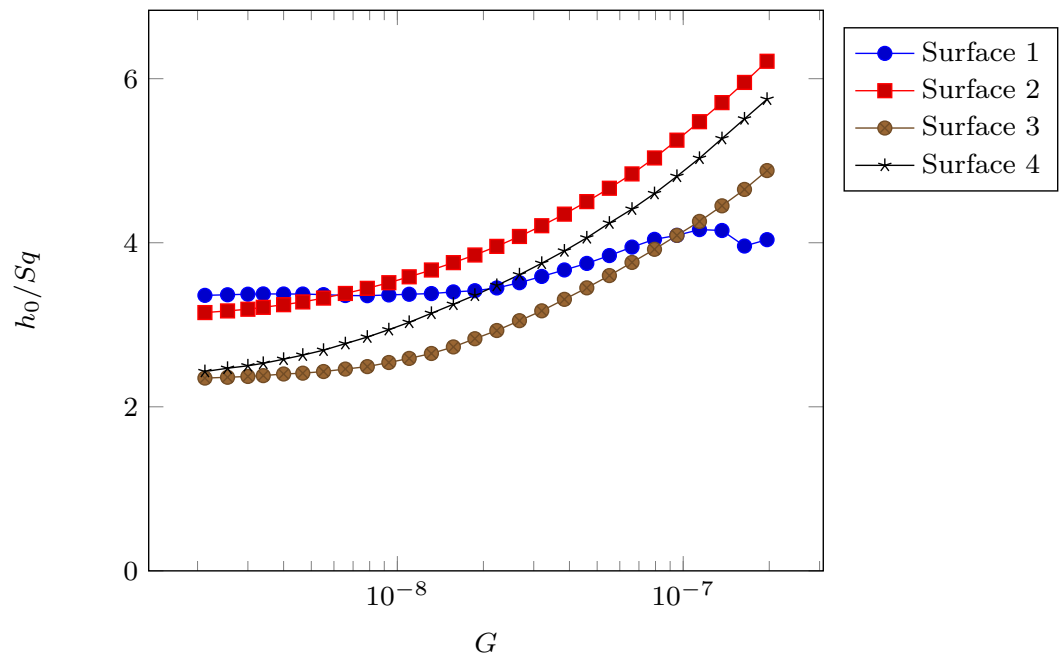

Fig. 3 Average film thickness as a function of the duty parameter

Figure 6 presents the pressure distribution obtained with the four surfaces at the maximum value of $G$ where the hydrodynamic load generation is maximum. First, the pressure map varies from one surface to the other because of 


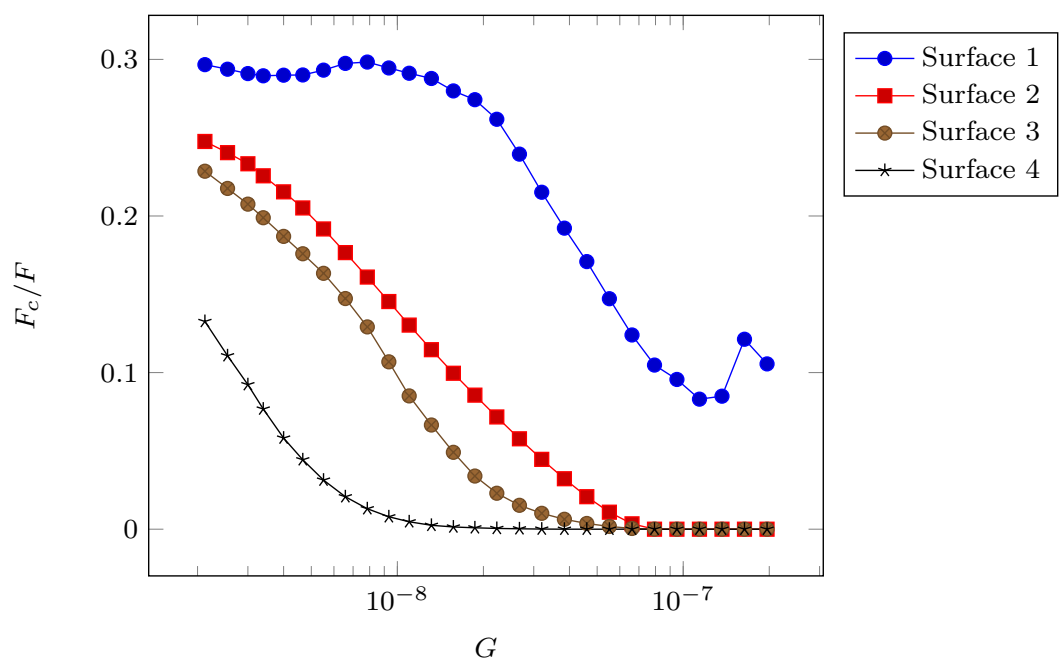

Fig. 4 Dimensionless contact force as a function of the duty parameter

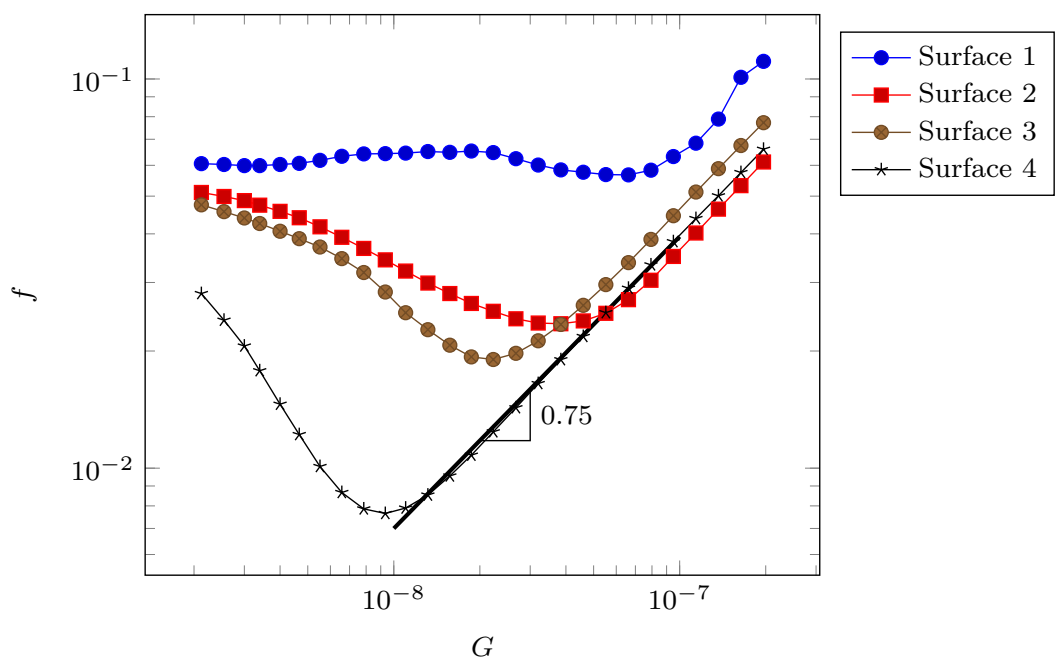

Fig. 5 Friction coefficient as a function of the duty parameter

the difference in local height distribution. The film height variations lead to pressure spikes and also pressure decreases, where cavitation can occur (blue areas on the figure). An interesting point is that the characteristic wavelength of the pressure distribution is much bigger than the roughness local wavelength (see figure 2). This indicates that the asperities do not act as an isolated single bearing, as proposed by So and Chen [31] but that a collective effect exists, which will be analyzed in the present paper. 


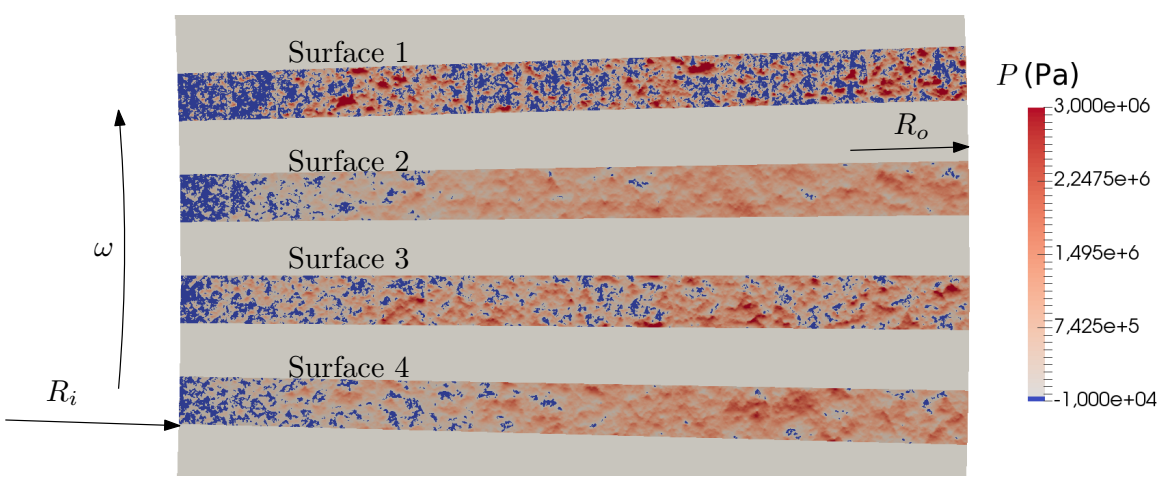

Fig. 6 Pressure distribution for $G=1.96 \times 10^{-7}$

\section{Analysis of the pressure build-up mechanism}

To identify the collective effect, the surfaces will be split in subsurfaces. The behavior of each subsurface will be analyzed to highlight any particular trends.

Figure 7 presents the principle of the surface split. Here, the domain is cut into 16 subdomains in the radial direction. At the radial boundaries of each sub-domain, a constant pressure $p_{0}$ is applied; whereas the periodic condition in the circumferential direction is preserved.

The results are computed as functions of the hydrodynamic parameter $\psi$ :

$$
\psi=\frac{6 \mu \omega R S q \sqrt{\lambda_{r} \lambda_{\theta}}}{\left(p_{0}-p_{\text {cav }}\right) h_{0}^{3}}
$$

where $R$ is the mean radius of the considered subsurface. The analyzed results are the dimensionless load $\bar{L}$ :

$$
\bar{L}=\frac{p_{a v g}-p_{0}}{p_{0}-p_{c a v}}
$$

and the dimensionless radial mass flow rate $\bar{m}$ :

$$
\bar{m}=\frac{h_{0} \dot{m}}{\rho \omega S q^{2} 2 \pi R^{2}}
$$

where $p_{\text {avg }}$ is the average pressure on the subsurface and $\dot{m}$ the radial mass flow rate.

The dimensionless variables were chosen to ensure that for a given $\psi$, the dimensionless load and mass flow rate remains unchanged - even if some dimensional parameters are varied.

Figure 8 presents the value of $\bar{m}$ and $\bar{L}$ on each subsurface of surface 4 when $\psi$ is set at 6.22 and $h / S q=10$ to avoid any asperity contact. Surface 4 is chosen because it provides the most typical Stribeck curve. First, the dimensionless load can be positive or negative depending on the surface. Negative load values are, however, limited by the occurrence of cavitation, which prevents very large negative pressure values. The differences observed on figure 8 could be due to 


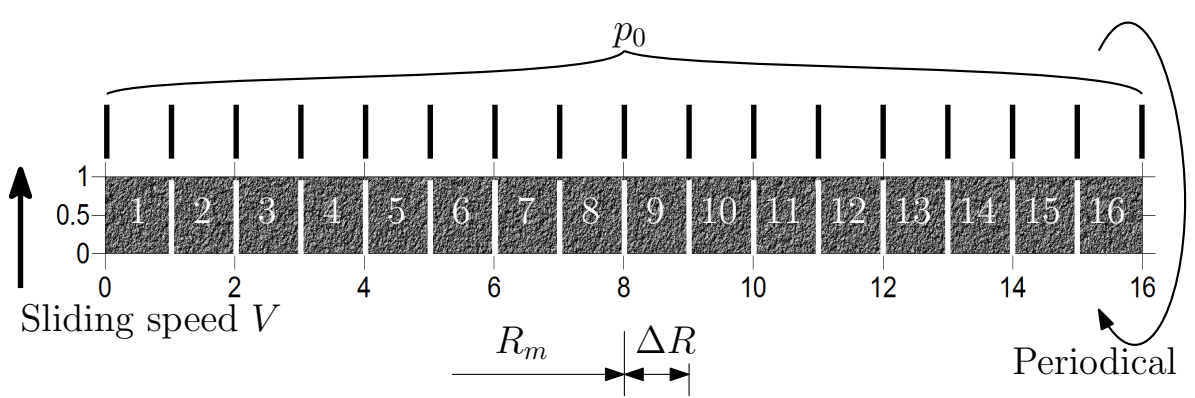

Fig. 7 Principle of subsurfaces analyzes

the differences in roughness distribution on each subsurface. However, it is difficult to use this result to explain the collective effect of roughness.

For each subsurface, the dimensionless flow also varies from positive to negative values. A positive sign corresponds to a flow from the left to the right of figure 7 and negative values to an opposite flow direction. The mass flow rate is generated by the sliding motion. The direction of the flow is due to the local difference in the roughness arrangement on each subsurface.

This last result is very interesting. When the subsurfaces are connected together, the mass flow rate must be the same on each subsurface to ensure mass conservation on the whole domain. Thus, the pressure at the boundaries of the subsurface must change to ensure mass flow conservation. Consequently, this offers a scenario for the collective roughness induced pressure generation mechanism.

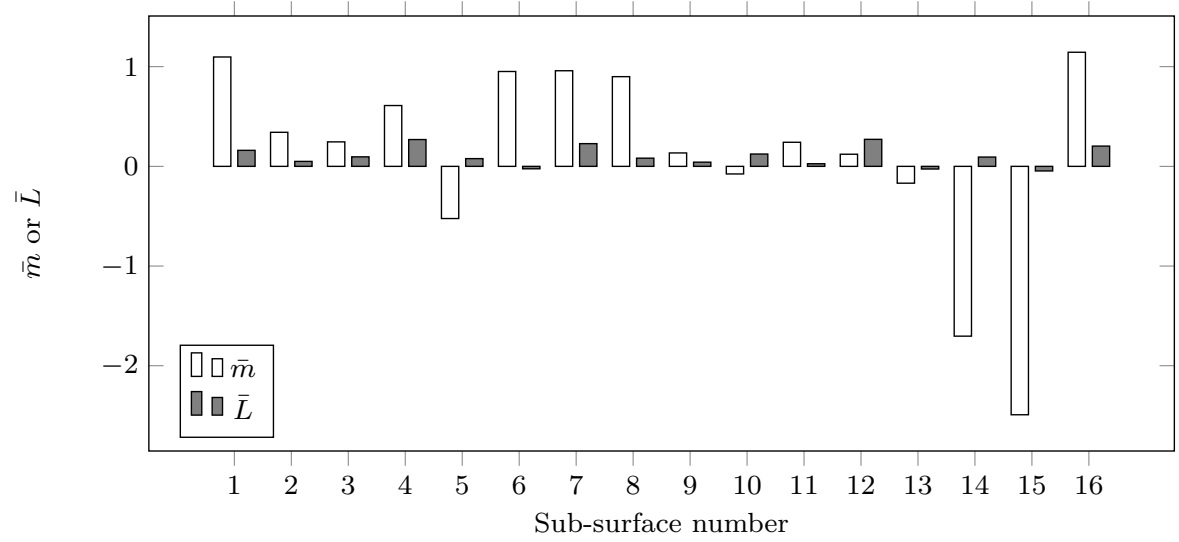

Fig. 8 Dimensionless leakage and load for each subsurface of surface 4 with $\psi=6.22$ and $h / S q=10$

Let us connect two subsurfaces. The roughness induced flow is now named pumping flow. For example, subsurfaces 1 and 2 should be connected. The 
pumping flow of subsurface 1 is positive and higher than the pumping flow of subsurface 2. To balance the flow rates, it is necessary to reduce the flow number 1 and increase the flow number 2. A possible solution is that a pressure flow from the center of the total domain is created, as illustrated on Fig 9 a). The net flow $\dot{m}$ on the subsurfaces must be equal:

$$
\dot{m}=\dot{m}_{p u 1}+\dot{m}_{p r 1}=\dot{m}_{p u 2}+\dot{m}_{p r 2}
$$

where $p u$ stands for the pumping flow and $p r$ for the pressure flow. If we assume that the pressure flow on the two subsurfaces are opposite:

$$
-\dot{m}_{p r 1}=\dot{m}_{p r 2}=\dot{m}_{p r}
$$

then it is possible to express this flow as a function of the pumping flow:

$$
\dot{m}_{p r}=\frac{\dot{m}_{p u 1}-\dot{m}_{p u 2}}{2}
$$

To ensure pressure generation between the two subsurfaces, it is necessary that $m_{p r}>0$. This condition is reached if:

$$
\dot{m}_{p u 1}>\dot{m}_{p u 2}
$$

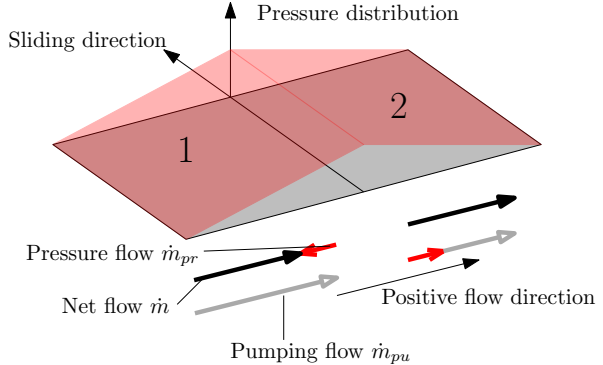

a)

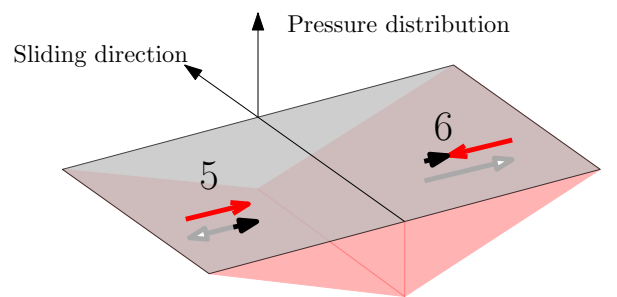

b)

Fig. 9 Pressure generation mechanism with a) subsurfaces 1 and 2 (positive effect), b) subsurfaces 5 and 6 (negative effect)

Using the same reasoning, when subsurfaces 5 and 6 are being connected, a negative pressure will be generated because $\dot{m}_{p u 5}<\bar{m}_{p u 6}$; as illustrated in Fig 9 b).

To check this theory, simulations have been performed after connecting subsurfaces 1 with 2 and 5 with 6 . The resulting pressure distributions are given in Fig 10 when $\psi=6.22$ and $h / S q=10$. The real pressure map is obviously not triangular shaped, as proposed in the theory, but it can be seen that more pressure is generated with subsurfaces 1 and 2 when compared to subsurfaces 5 and 6 , where more cavitation occurs. Average pressure profiles are presented on Fig 11. It can be clearly seen that the average pressure has the sign of $\dot{m}_{p u 1}-\dot{m}_{p u 2}$. This simple example confirms the proposed theory. 


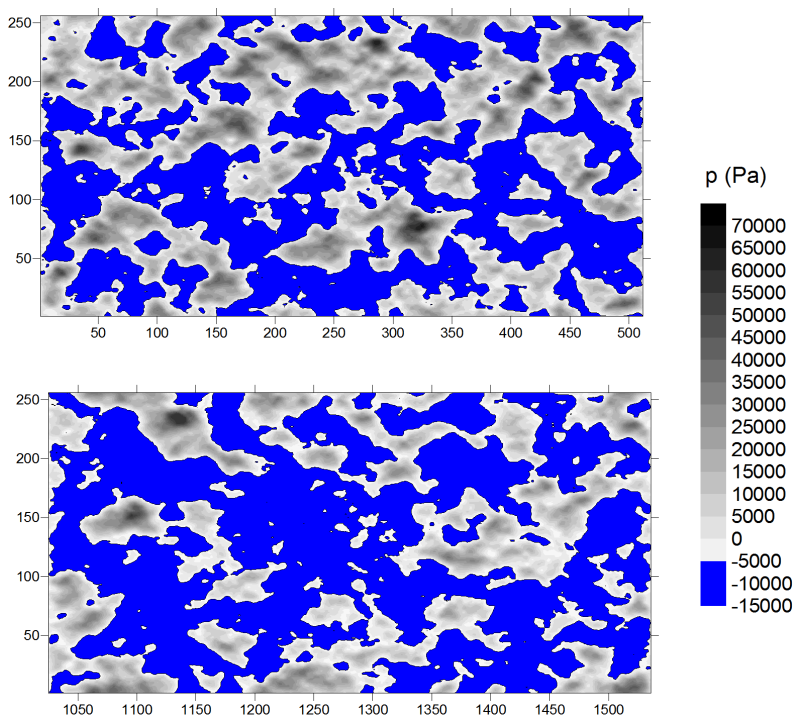

Fig. 10 Pressure distribution on subsurfaces 1 and 2 (top), and 5 and 6 (bottom) with $\psi=6.22$ and $h / S q=10$

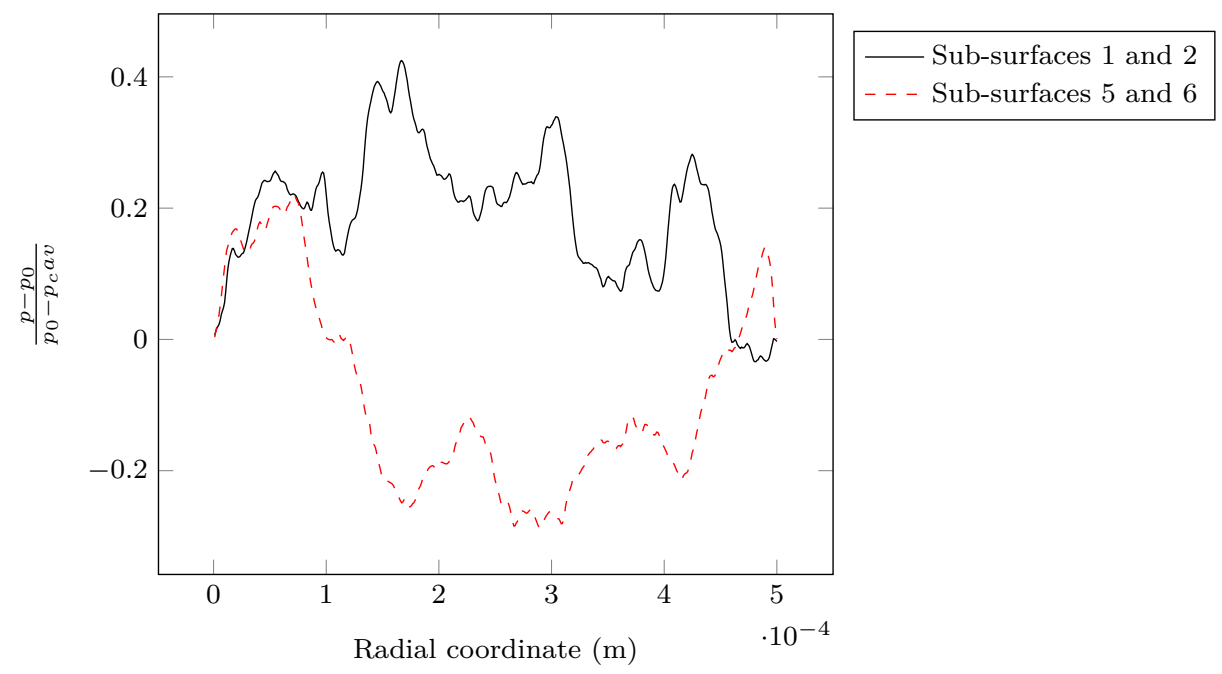

Fig. 11 Average pressure profiles with $\psi=6.22$ and $h / S q=10$

To continue the verification of the present theory, surfaces 1 to 4 (Fig 2) are used. They are first split in 16 subsurfaces, as explained in Fig 7, to calculate the dimensionless pumping flow rate $\bar{m}_{p u}$. They are then split into eight subsurfaces to compute the dimensionless load $\bar{L}$. According to the proposed theory, the following result should be found:

$$
\bar{L}_{i, i+1} \propto \bar{m}_{p u i}-\bar{m}_{p u i+1}
$$


where $i$ and $i+1$ refer to the two sets of 16 subsurfaces, constituting the eighth subsurfaces on which the load is computed. The results are presented in figure 12 a) for $\psi=6.22$ and $h / S q=10$. The results exhibit a tendency confirming the proposed theory. An increase in the pumping flowrate difference leads to an increase of the generated load. However, because of the non-linear behavior induced by cavitation occurrence, the proportionality of the parameters cannot be seen. If the hydrodynamic parameters $\psi$ is reduced to values where cavitation does not occur, then a better correlation is obtained; as can be seen in Fig 12 b).
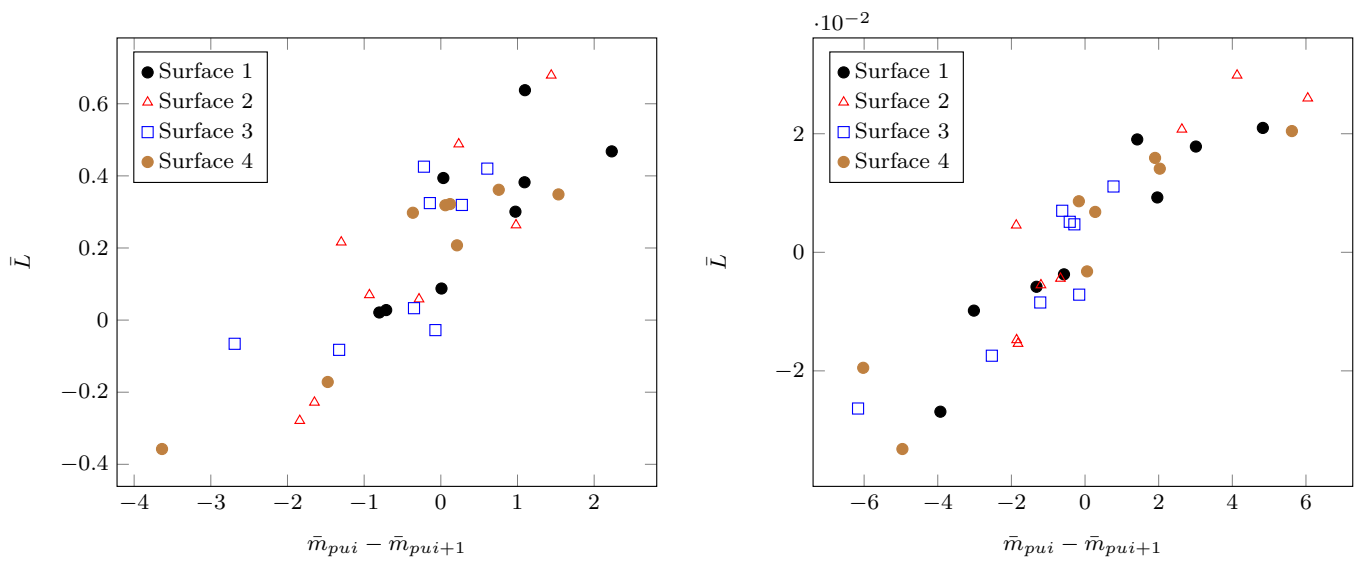

Fig. 12 Relation between the generated load and the pumping flow rate difference with $h / S q=10$ and a) $\psi=6.22$ b) $\psi=0.166$

This first analysis allows us to propose a mechanism of the collective roughness induced pressure between parallel surfaces. The local difference of the roughness distribution can create lateral flow of the fluid. To ensure balance of the flow it is necessary that some additional pressure flows exist. Thus, a pressure build-up takes place because of the difference in the lateral pumping flow rate from place to place.

This theory raises several questions: 1 - why do the pumping flows not generate negative loads ? 2 - Why is the random character of pumping distribution necessary ? 3 -What is causing the randomness of pumping flows? Possible answers to these questions can be formulated:

1. As shown in Fig. 10, an unfavorable pumping flow rate distribution will promote cavitation. Cavitation occurrences will avoid reaching highly negative pressures, whereas in other places, where the pumping flow is better distributed build-up pressure will occur. Finally a positive net force can be generated. It will be shown in the next section that the pumping flow rate will vanish when the considered area is cavitated (see eq. 16), reducing thus the undesirable effect promoted by negative flow rate difference (see eq. 11). 
2. According to eq. 11, if all the subsurfaces exhibit the same pumping flow rates, it would not be possible to create an additional hydrodynamic fluid force. Thus, a non-uniform pumping flow rate distribution is necessary. A random distribution fulfills this condition. However, we can imagine that by soundly choosing the pumping flow rates, the load generation could be more efficient than with a random distribution. This principle was, for example, used by Adjemout et al. [?] to create efficient surface texturation with triangle in opposite orientation. A random distribution of the pumping flow rate is a way to generate hydrodynamic pressure but not the best.

3. The statistical properties of the rough surfaces are calculated on the whole domain. The subsurfaces extracted from the original surface do not exactly exhibit the same statistical properties. In addition, the location of the peaks and valleys is not the same on all the subsurfaces. These differences can explain the random character of the pumping flow rate.

In addition, it is worth noting that the whole analysis on pumping flow rate was performed with zero pressure difference at the domain boundaries (see Fig. 7) contrary to the standard seal operation (see Fig. 1). It means that a pressure difference is not necessary to generate the additional hydrodynamic pressure in the fluid film.

\section{Simplified modeling of the pressure generation}

In this section, we propose to develop a very simple model of the pressure build-up mechanism that will be compared to the deterministic solution presented at the beginning of the paper. It is an axisymmetrical model based on the mass conservation in the radial direction. It must thus consider the pressure flow and the pumping flow as indicated in equation (7). The pressure flow can be expressed with usual approaches, such as flow factor. However, it is necessary to propose a model for the pumping flow rate.

\subsection{Analysis of the pumping flow rate}

Figure 13 presents the evolution, for eight representative subsurfaces of surface 4 , of the dimensionless pumping flow rate as a function of the hydrodynamic parameter $\psi$. Whatever the subsurface considered, for low $\psi$ values, the dimensionless flow rate is first constant and then its magnitude tends to decrease to zero when the hydrodynamic parameter is increased. The decrease of the flow rate corresponds to the appearance of cavitation in the domain. Let us name $\bar{m}_{0}$ the constant value reached at low $\psi$ values.

The values of $\bar{m}_{0}$ have been calculated for 512 subsurfaces extracted from surfaces 1 to 4 (each of them being split in 128 subsurfaces). This large number has been choose to obtain a representative distribution. An histogram of the $\bar{m}_{0}$ distribution values is presented in figure 14 , which exhibits a bell shape 


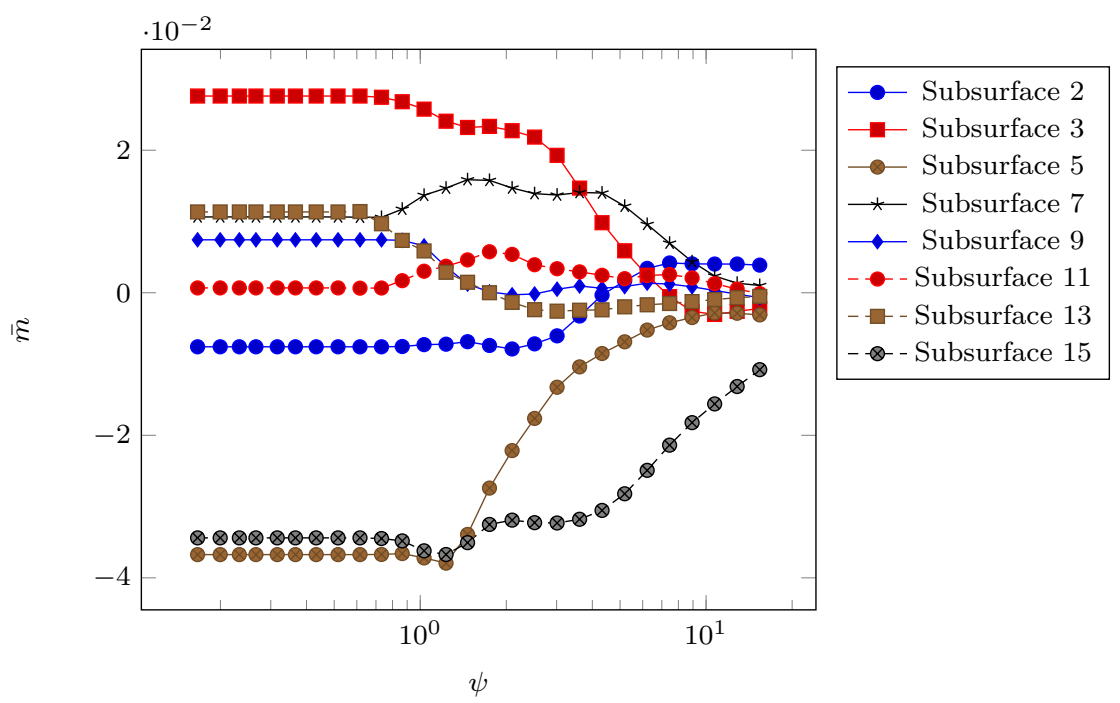

Fig. 13 Dimensionless pumping rate as a function of the hydrodynamic parameter, $\psi$ for eight subsurfaces of surface 4

that can be well fitted with a Gaussian curve $f$ centered on zero:

$$
f\left(\bar{m}_{0}\right)=\frac{1}{\sigma \sqrt{2 \pi}} e^{-\frac{\bar{m}_{0}^{2}}{\sigma^{2}}}
$$

$\sigma$ is the standard deviation of the distribution. The zero average of the distribution is not surprising because there is no reason why one flow direction would be preferred.

By using equations 7 and 9, it can be shown that the net flow obtained when connecting two subdomains would be:

$$
\dot{m}=\frac{\dot{m}_{p u 1}+\dot{m}_{p u 2}}{2}=\dot{m}_{p u 1+2}
$$

This net flow is, according to our definition, the pumping flow of the subsurface composed of subsurfaces 1 and 2 . If subsurfaces 1 and 2 have a lateral size $\Delta R$ with a standard deviation $\sigma$ on the mass flow rate distribution, then it can be shown that, because of relation 13, the standard deviation for subsurfaces of size $2 \Delta R$ is $\frac{\sigma}{\sqrt{2}}$. This relation works if the values of neighboring surfaces are uncorrelated. Consequently, it is possible to express the dependence of the standard deviation to the subsurface lateral size:

$$
\sigma \propto \frac{1}{\sqrt{\Delta R}}
$$

The actual standard deviation of the pumping flow rate distribution has been calculated for subsurfaces of different sizes (from $\frac{1}{8}$ to $\frac{1}{128}$ of the total surface). The results are presented in figure 15. The expected relation 14 is recovered 


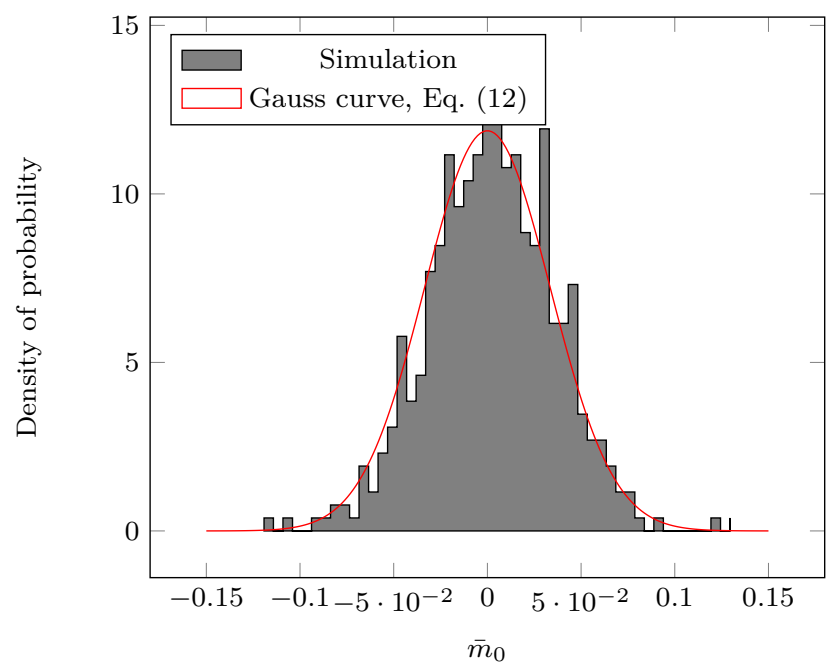

Fig. 14 Distribution of $\bar{m}_{0}$ for subsurfaces corresponding to $\frac{1}{128}$ of the full surfaces

for high values of the domain size. However, an asymptotic value of 0.0336 is reached when the domain size is decreased. The deviation from the theoretical relation 14 appears when the domain size $\Delta R$ is decreased to values of the order of magnitude of the asperities correlation length $\lambda_{r}$. Below this value, the neighboring surfaces are logically correlated, leading to a breakdown of the theoretical relation. The evolution of $\sigma$ can be fitted with the following relation:

$$
\sigma=\frac{0.0336}{\left[1+\left(0.1 \frac{\Delta R}{\lambda_{r}}\right)^{4}\right]^{\frac{1}{8}}}
$$

Finally, it is necessary to model the evolution of $\bar{m}$ with the hydrodynamic parameter $\psi$. This evolution has been averaged on the subsurfaces extracted from surfaces 1 to 4 and the results are presented in figure 16. It was found that a good approximation is given by:

$$
\bar{m}=\frac{\bar{m}_{0}}{\left[1+\left(\frac{\psi}{3.7}\right)^{2.5}\right]^{\frac{1}{2.5}}}
$$

At high $\psi$ values, the mass flow rate is proportional to $\psi^{-1}$. The grey area represents the values obtained when the averaging is performed only on the subsurfaces extracted from a given surface $(1,2,3$ or 4$)$. In addition to the random value of $\bar{m}_{0}$, there are also some different evolutions with $\psi$ due to the local difference in roughness distribution; however, this point will not be addressed here. 


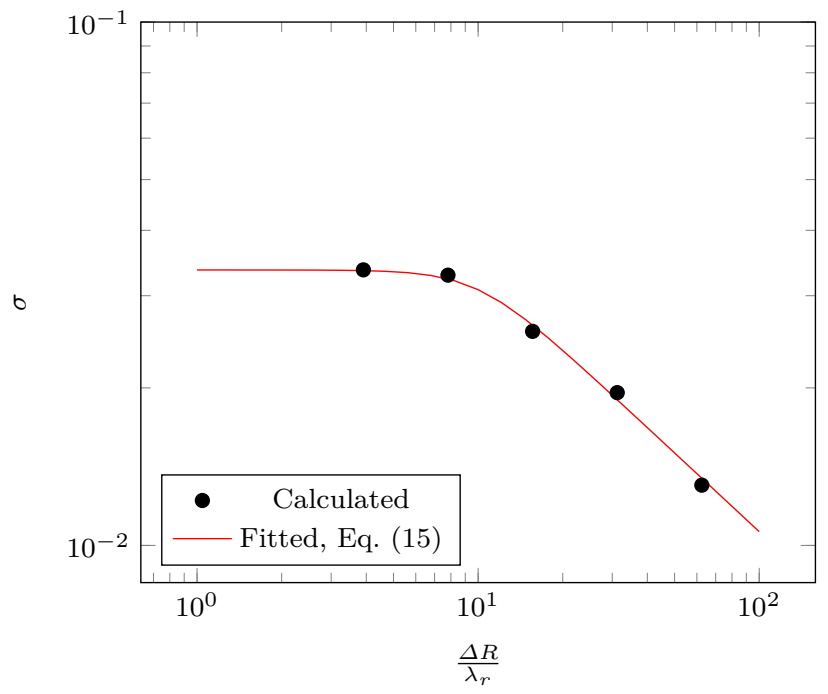

Fig. 15 Variation of the standard deviation of the $\bar{m}_{0}$ distribution with the size of the subsurface

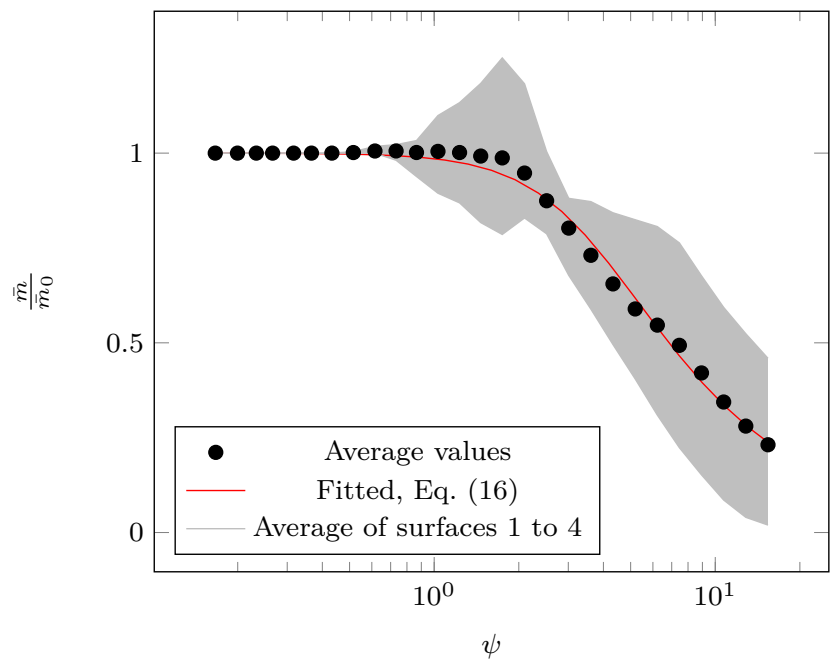

Fig. 16 Average evolution of the mass flow rate $\frac{\bar{m}}{\bar{m}_{0}}$ as a function of the hydrodynamic parameter $\psi$

\subsection{Simplified model}

The problem is supposed to be axisymmetrical and stationary. The objective of the model is to calculate the average pressure $\bar{p}$ and not the local pressure distribution $p$, as with a deterministic model. At a given radius $r$, the net flow is the sum of the pressure flow and the pumping flow as previously discussed 
(see equation (7)). The pumping flow is given by equation 6 :

$$
\dot{m}_{p u}=2 \pi r \rho \omega r \frac{S q^{2}}{h_{0}} \bar{m}(r)
$$

The pressure flow can be expressed using the flow factors theory $[28,27]$ :

$$
\dot{m}_{p r}=-2 \pi \phi_{r} \frac{\rho h_{0}^{3}}{12 \mu} \frac{\partial \bar{p}}{\partial r}
$$

According to Harp and Salant [12], the pressure flow factor is not affected by the micro-cavitation occurring at the asperity level. Thus, it is only possible to express this factor as a function of the geometrical parameters. The solution proposed by Tripp [33] is used in the present work.

Given that the problem is stationary, the net mass flow is invariant along the $r$ direction. Therefore, the following Reynolds equation is obtained:

$$
\frac{\partial}{\partial r}\left(\phi_{r} \frac{\rho h_{0}^{3}}{12 \mu} \frac{\partial \bar{p}}{\partial r}\right)=\frac{\partial}{\partial r}\left[\rho r^{2} \omega \frac{S q^{2}}{h_{0}} \bar{m}(r)\right]
$$

The originality of the present approach is that the pumping term varies along the $r$ direction and is not vanished by averaging, which is usually done when the flow factor theory is used. Knowing the rough surface parameters and the mesh lateral size $\Delta R$, we are able to calculate the standard deviation $\sigma$ using equation 15. Then, a Gaussian random series is generated with the prescribed standard deviation. A value of $\bar{m}_{0}$ from this series is attributed at each node. The Reynolds equation is then solved in an iterative process. The value of $\bar{m}$ is updated at each step using equation 16 , knowing the $\psi$ value from the calculated pressure.

Given that at low $G$ values an asperity contact is expected to occur, it is necessary to consider the asperity contact pressure $p_{c}$. The simplified Greenwood and Williamson model [8] assuming an exponential distribution is used:

$$
p_{c}=E^{\prime} \frac{S q}{8} \sqrt{\frac{\pi b^{3}}{8 \lambda_{r} \lambda_{\theta}}} \exp \left(\frac{2 a}{b}\right) \exp \left(-\frac{2 h}{b S q}\right)
$$

where $E^{\prime}$ is the composite elastic modulus of the rotor and stator materials. $a$ $=1$ and $b=0.8$ are fitting parameters, which were chosen to provide a contact pressure corresponding to the average response of surfaces 1 to 4 .

As in the previous simulations, the distance $h_{0}$ between the two rings is adjusted to ensure balance between the closing force and the opening force due to the fluid pressure and asperity contact. 


\subsection{Results}

A total of 10 different random series were generated and introduced in the model. Figures 17 to 19 present the film thickness, contact force and friction coefficient evolution for the 10 different series when the duty parameter is varied. A grey fill area represents the limit of the results, as given by the deterministic simulations presented at the beginning of this paper.

The film thickness obtained at low $G$ values with the simplified model is almost independent of the series used (figure 17). This is not surprising because the hydrodynamic pressure generation is not significant at low speed, and the film thickness is thus controlled by the asperity contact. By checking figure 18 , it is confirmed that almost all the series provide the same contact force, contrary to the deterministic solution.

When the duty parameter is increased, in most of the cases the film thickness increases, the contact force decreases and the friction coefficient decreases, reaches a minimum and then increases. When the surfaces are fully separated, the 0.75 slope coefficient on the friction coefficient is obtained as with the deterministic solution. The simplified model is able to simulate the roughness induced lift-off and in 7 cases among 10 gives results that are in good agreement with the deterministic solutions; that is, the results are within the grey area. The three other cases corresponds to thinner film, higher contact force and higher friction force. It is not surprising that the simplified model provides a higher variability of the results because it is a one dimensional approach, which is thus more sensitive to the initial random distribution of $\bar{m}_{0}$. Moreover, the deterministic computations were limited to four cases because of the computation time, which is 6000 times longer than with the simplified model. A simulation on more surfaces would extend the grey area. In addition, the extension of the simplified model to two dimensions would probably reduce the dependency to the random series used and allow us to provide a more realistic two dimensional average pressure distribution.

Figure 20 shows the average pressure profiles at two different values of the duty parameter $G$, obtained with series 5 (left) and series 8 (right), which provide the highest and lowest friction values, respectively. In the case of series 5 , when the duty parameter is increased, some hydrodynamic pressure spikes are created close to the outer radius whereas the area close to the inner radius exhibits a pressure close to the atmospheric pressure. The random distribution of the pumping flow rate, in this case, tends to dry up the contact and this leads to low fluid force and high friction. In contrast, series 5 leads to a global increase of the fluid pressure level in the contact, when $G$ is increased, which promotes surface separation and low friction.

\section{Conclusion}

The separation observed when two parallel surfaces are in relative sliding motion in presence of a lubricant has only recently been fully simulated thanks to 


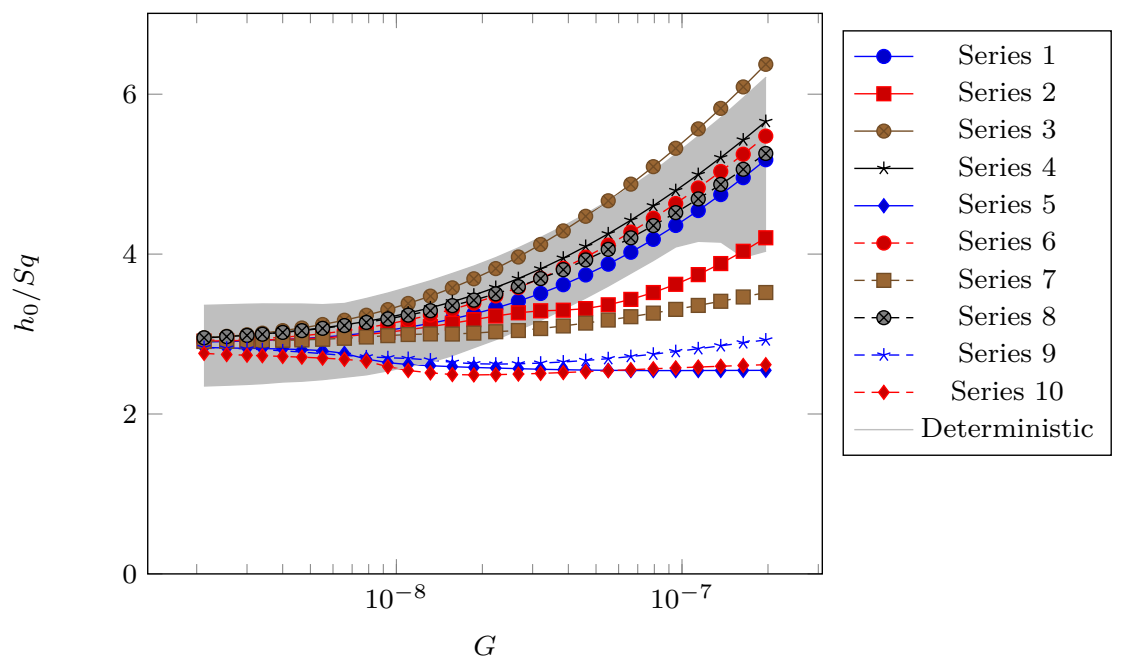

Fig. 17 Comparison of the average film thickness calculated with the simplified model to the deterministic simulations

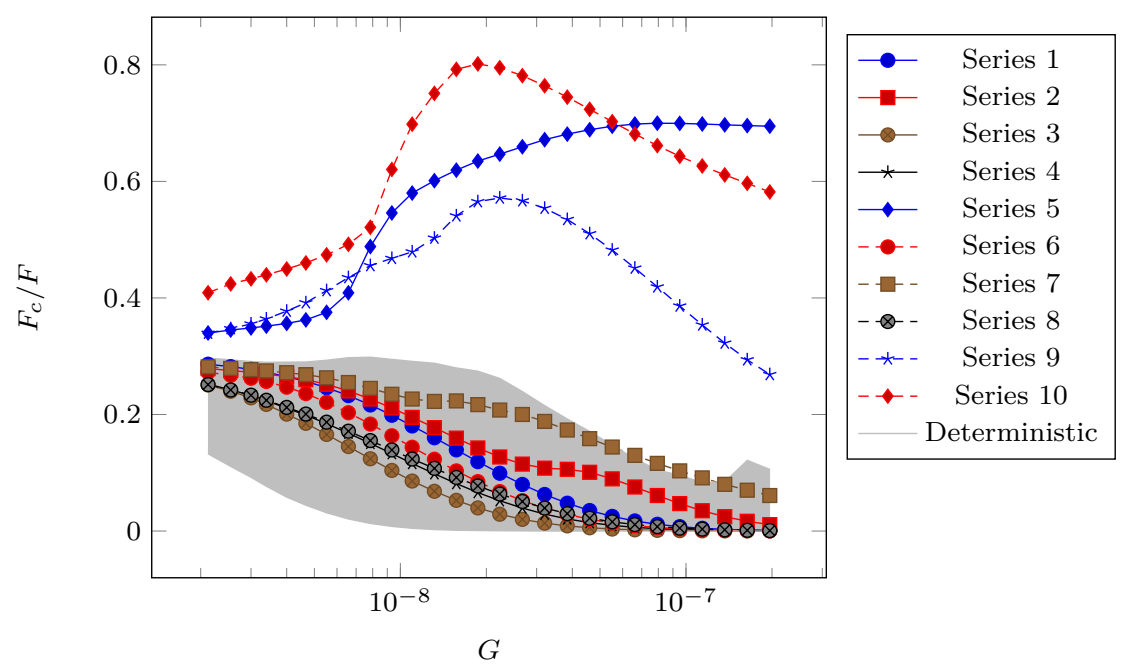

Fig. 18 Comparison of the contact force calculated with the simplified model to the deterministic simulations

deterministic models involving very fine meshes that are able to capture the surface topography.

The hydrodynamic pressure generation is due to the height variations that are provided by the roughness. The asperities do not act as an independent slider bearing but instead exhibit a collective effect on the pressure distribution. Indeed, a transverse pumping mechanism that varies from place to place with the surface topography allows a global pressure build-up to be generated by the difference in transverse pumping flows. The generated load appears 


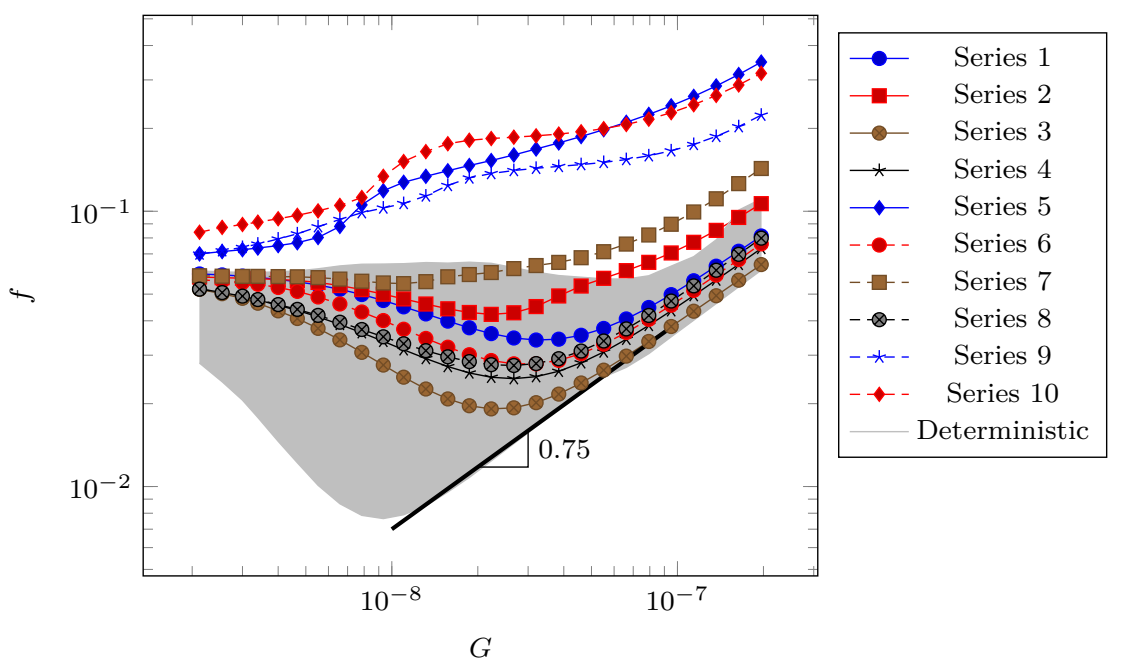

Fig. 19 Comparison of the friction coefficient calculated with the simplified model to the deterministic simulations
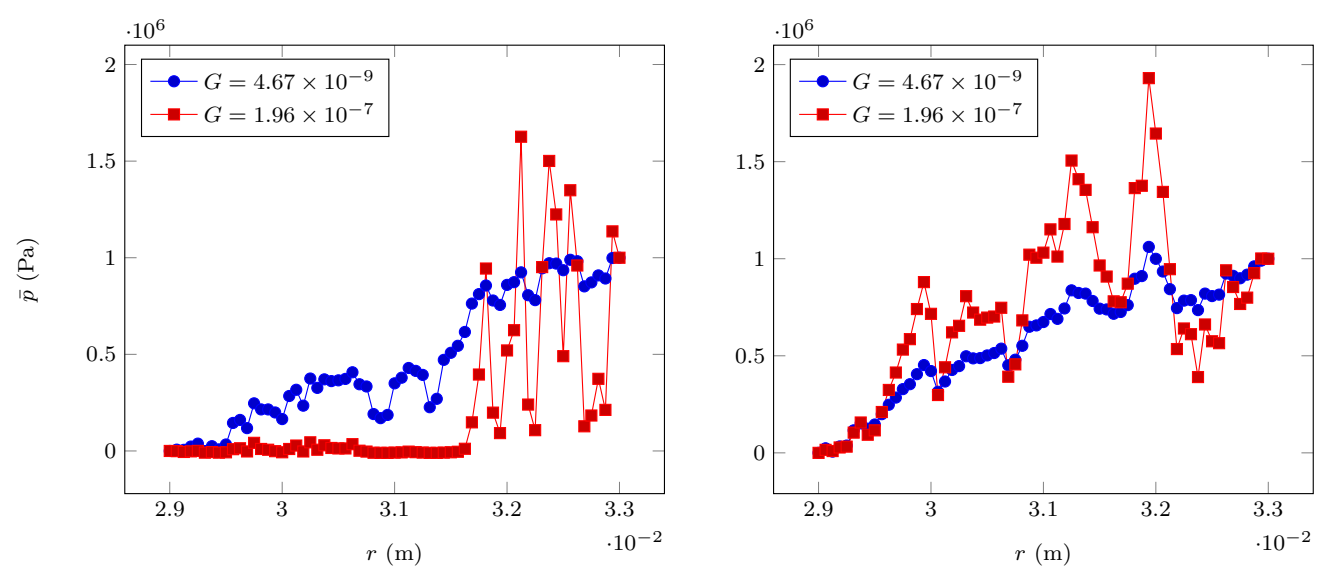

Fig. 20 Average pressure profiles obtained with series 5 (left) and series 8 (right) at different $G$ values

to be proportional to the difference in pumping flow rate from neighboring surfaces.

Based on this observation, a simplified model is proposed to include the random transverse pumping and to simulate the roughness induced pressure generation in a more computational efficient way than the deterministic simulations. In contrast to the usual models (flow factor theory or homogenization), where all the roughness effects are averaged, we show that is is necessary to consider the random nature of some parameters to be able to simulate the roughness induced pressure. The results that we have obtained are in clear 
correlation with those given by the deterministic model. However, this simplified approach needs to be improved and extended to two-dimensional flows. 


\section{References}

1. Ayadi, K., Brunetière, N., Tournerie, B., Maoui, A.: Experimental and numerical study of the lubrication regimes of a liquid mechanical seal. Tribology International 92, 96 - 108 (2015). DOI http://dx.doi.org/10.1016/j.triboint.2015.05.022. URL http://www.sciencedirect.com/science/article/pii/S0301679X15002212

2. Bayada, G., Faure, J.: A double scale analysis approach of the reynolds roughness comments and application to the journal bearing. Journal of Tribology 111(2), 323$330(1989)$

3. Bayada, G., Martin, S., Vasquez, C.: An average flow model of the reynolds roughness including a mass-flow preserving cavitation model. Journal of Tribology 127(4), 797-802 (2005)

4. Brunetière, N., Tournerie, B., Minet, C.: On the roughness induced hydrodynamic pressure in mechanical face seals. In: 21st International Conference on Fluid Sealing, pp. 103-113. BHRGroup, Milton Keynes, UK (2011)

5. Denny, D.: Some measurements of fluid pressures between plane parallel thrust surfaces with special reference to radial-face seals. Wear 4, 64-83 (1961)

6. Flitney, R., Nau, B.: Performance testing of mechanical seals. In: B. Nau (ed.) 13th International Conference on Fluid Sealing, pp. 441-466. BHR Group, Kluwer Academic Publishers, Brugge, Belgium (1992)

7. Francisco, A., Bruneti $\widetilde{A}^{*}$ re, N.: A hybrid method for fast and efficient rough surface generation. IMechE, Part J, Journal of Engineering Tribology 230(7), 747-768 (2016). DOI 10.1177/1350650115612116

8. Greenwood, J.A., Williamson, J.B.P.: Contact of nominally flat surfaces. Proceedings of the Royal Society (London) A295, 300-319 (1966)

9. Gropper, D., Wang, L., Harvey, T.J.: Hydrodynamic lubrication of textured surfaces: A review of modeling techniques and key findings. Tribology International 94, 509 - 529 (2016). DOI http://dx.doi.org/10.1016/j.triboint.2015.10.009. URL http://www.sciencedirect.com/science/article/pii/S0301679X15004582

10. Hamilton, D., Walowit, J., Allen, C.: A theory of lubrication by micro-irregularities. Journal of Basic Engineering 88, 177-185 (1966)

11. Hamrock, B., Dowson, D.: Ball Bearing Lubrication - The Elastohydrodynamics of Elliptical Contacts. Wiley, New York, USA (1981). DOI 047103553X

12. Harp, S., Salant, R.: An average flow model of rough surface lubrication with interasperity cavitation. Journal of Tribology 123(1), 134-143 (2001)

13. Hill, I., Hill, R., Holder, R.: Fitting johnson curves by moments. Applied Statistics 25, 180-189 (1976)

14. Hu, Y., Tonder, K.: Simulation of 3-d random rough surface by 2-d digital filter and fourier analysis. International Journal of Machine Tools and Manufacture 32(1/2), 83-90 (1992)

15. Kane, M., Bou-Saïd, B.: Comparison of homogenization and direct techniques for the treatment of roughness in incompressible lubrication. Journal of Tribology 126(4), 733-737 (2004)

16. Lebeck, A.: Parallel sliding load support in the mixed friction regime. part 1 - the experimental data. Journal of Tribology 109(1), 189-195 (1987)

17. Lebeck, A.: Parallel sliding load support in the mixed friction regime. part 2 - evaluations of the mechanisms. Journal of Tribology 109(1), 196-205 (1987)

18. Lebeck, A.: Principle and Design of Mechanical Face Seals. John Wiley \& Sons, Inc., New York (1991)

19. Lebeck, A.: Mixed lubrication in mechanical face seals with plain faces. IMechE, Part J, Journal of Engineering Tribology 213(J3), 163-175 (1999)

20. Lubbinge, H.: On the lubrication of mechanical face seals. Ph.D. thesis, University of Twente, Netherlands (1999)

21. Minet, C., Brunetière, N., Tournerie, B.: A deterministic mixed lubrication model for mechanical seals. Journal of Tribology 133(4), 042203 (11 pages) (2011). DOI $10.1115 / 1.4005068$

22. Minet, C., Brunetière, N., Tournerie, B.: On the lubrication of mechanical seals with rough surfaces: A parametric study. IMechE, Part J, Journal of Engineering Tribology 226(12), 1109-1126 (2012) 
23. Nau, B.: Hydrodynamic lubrication in face seals. In: BHRA (ed.) 3rd International Conference on Fluid Sealing, Paper E5, pp. 73-120. BHRA, Cambridge, England (1967)

24. Nau, B.S.: Hydrodynamics in face seal films. In: 2 nd International Conference on Fluid Sealing, paper F5, pp. 61-80. BHRA, Cranfield, England (1964)

25. Nyemeck, A., Brunetiï $\frac{1}{2}$ re, N., Tournerie, B.: A multiscale approach to the mixed lubrication regime: Application to mechanical seals. Tribology Letters 47(3), 417-429 (2012)

26. Pape, J.: Fundamental research on a radial face seal. ASLE Transactions 11(4), 302-309 (1968)

27. Patir, N., Cheng, H.: An average flow model for determining effects of three-dimensional roughness on partial hydrodynamic lubrication. Journal of Lubrication Technology 100(1), 12-17 (1978)

28. Patir, N., Cheng, H.: Application of average flow model to lubrication between rough sliding surfaces. Journal of Lubrication Technology 101(2), 220-230 (1979)

29. Reynolds, O.: On the theory of lubrication and its application to mr. beauchamp tower's experiments, including an experimental determination of the viscosity of olive oil. Philosophical Transactions of the Royal Society of London 177, 157-234 (1886)

30. So, H., Chen, C.: Effects of micro-wedges formed between parallel surfaces on mixed lubrication - part i: Experimental evidence. Tribology Letters 17(3), 513-520 (2004). DOI 10.1023/B:TRIL.0000044499.67099.74. URL https://doi.org/10.1023/B:TRIL.0000044499.67099.74

31. So, H., Chen, C.: Effects of micro-wedges formed between parallel surfaces on mixed lubrication - part ii: modeling. Tribology Letters 19(2), 83-91 (2005). DOI 10.1007/s11249-005-5083-6. URL https://doi.org/10.1007/s11249-005-5083-6

32. Summers-Smith, D.: Laboratory investigation of the performance of a radial face seal. In BHRA (ed.) 1st International Conference on Fluid Sealing, paper D1. BHRA, Ashford, UK (1961)

33. Tripp, J.: Surface roughness effects in hydrodynamic lubrication: The flow factor method. Journal of Lubrication Technology 105(3), 458-465 (1983)

34. Vezjak, A., Vizintin, J.: Experimental study on the relationship between lubrication regime and the performance of mechanical seals. Lubrication Engineering 57(1), 17-22 (2001)

35. Watson, W., Spedding, T.: The time series modelling of non-gaussian engineering processes. Wear 83, 215-231 (1982) 